\title{
Linx
}

Revue des linguistes de l'université Paris X Nanterre

47 | 2002

Du sens au sens

\section{Petites rêveries littérales sur les noms de quelques personnages de Molière}

Reveries on the names of some of Molière's characters

\section{Michel Arrivé}

\section{(2) OpenEdition}

\section{Journals}

Édition électronique

URL : http://journals.openedition.org/linx/583

DOI : $10.4000 /$ linx.583

ISSN : 2118-9692

Éditeur

Presses universitaires de Paris Nanterre

\section{Édition imprimée}

Date de publication : 1 décembre 2002

Pagination : 65-77

ISSN : 0246-8743

\section{Référence électronique}

Michel Arrivé, "Petites rêveries littérales sur les noms de quelques personnages de Molière », Linx [En ligne], 47 | 2002, mis en ligne le 01 juin 2003, consulté le 01 mai 2019. URL : http:// journals.openedition.org/linx/583 ; DOI : 10.4000/linx.583 


\title{
Petites rêveries littérales sur les noms de quelques personnages de Molière
}

\author{
Michel Arrivé, Université Paris X - Nanterre
}

Je ne suis pas spécialiste de Molière. Je n’appartiens même pas à la glorieuse cohorte des «littéraires ». C'est donc à mes risques et périls que je me hasarde timidement sur un terrain qui n'est, en principe, pas le mien. Les risques? Ils sont réels : le principal est lié à la connaissance insuffisante de la bibliographie. Il y a, me dit un spécialiste reconnu, plus de 10000 travaux consacrés à Molière. Il n'est pas impossible que les petites rêveries auxquelles je me suis laissé aller aient déjà été faites par tel thésard de l'Arkansas (ou de Besançon...) ou dans tel articulet d'une revue néo-zélandaise (ou poitevine...). J'ai pris quelques précautions, certes : les éminents spécialistes que j'ai consultés m'ont fait part - non sans les précautions d'usage - de l'absence assez générale de curiosité pour l'onomastique moliéresque. L'article de Jean Serroy (2000) - à ma connaissance le seul parmi les productions récentes à prendre pour objet spécifique et exclusif quelques noms propres moliéresques - ne cite que les éditions, leurs notes (souvent bien décevantes), les dictionnaires de l'époque et quelques textes littéraires, contemporains, antérieurs ou postérieurs. Les quelques sondages que j'ai faits sur différents sites Internet m'ont pour l'essentiel laissé sur ma faim. Mais le risque subsiste, sournois.

À vrai dire, mes curiosités étaient au départ très limitées : quelques-uns des noms propres de trois pièces: Le Misantrope $e^{1}$, George $e^{2}$ Dandin, Le Bourgeois gentilhomme. Toutefois, dans l'espoir de trouver quelques éléments de confirmation à mes modestes spéculations, j'ai fait l'effort de constituer un inventaire exhaustif - au moins dans mes intentions - des noms de personnages de l'ensemble des pièces de Molière. Faute de place, cet inventaire ne peut être reproduit ici.

Je dois l'avouer: la consultation de cette longue liste n'a apporté à mes hypothèses de départ sur les noms que j'avais retenus ni confirmation nette, ni infirmation décisive. Tout au plus, sur deux points - j’y reviendrai - l'ombre d'une indication.

\footnotetext{
${ }^{1}$ On sait que c'est cette graphie, sans $-h-$, qui apparaît dans le titre de l'édition originale.
}

${ }^{2}$ Sans $-s$ finale, j'y reviendrai. 
Michel Arrivé

Cependant, l'inventaire en lui-même donne lieu à quelques remarques qui, pour être faciles, n'en sont pas moins intéressantes. Voici celles qui me paraissent les moins négligeables.

1. L'inventaire des noms propres moliéresques est assez peu abondant: 282 formes différentes pour 33 pièces $^{3}$. Mais qu'on y prenne garde : cet inventaire des noms propres ne correspond pas à celui des personnages. D'une part certains personnages - généralement très fugitifs - ne bénéficient pas d'un nom propre : ils n'apparaissent pas dans mon inventaire ${ }^{4}$. On constate d'autre part que 34 noms sont communs à des personnages apparaissant dans des pièces différentes : s'il n'y a que deux Anselme, Ariste et Dorimène, il y a trois Angélique comme trois Cléante, quatre Clitandre et même sept Sganarelle et autant de Valère. En sorte que le nombre des personnages s'élève finalement à 340 - ce qui est plus compatible avec le nombre des pièces : elles ont en effet un peu plus de 10 personnages (précisément 10,3) en moyenne ${ }^{5}$. Il serait naturellement intéressant de poser la question des éventuels traits communs entre les personnages qui, dans des pièces différentes, portent le même nom. Travail que je laisse à d'autres ${ }^{6}$, non sans soupçonner que les résultats seraient assez différents selon les noms. On pourrait par exemple se demander s'il n'y a pas un seul nom Sganarelle porté par le même (?) personnage revenant dans plusieurs pièces. Inversement, la pluralité des sept Valère - ou de tel nombre d'entre eux - serait peutêtre retenue.

2. Il est aisé de faire un classement sommaire des noms propres de l'inventaire. Ils se répartissent assez commodément entre les classes suivantes :

2.1. Accèdent au statut de noms propres un certain nombre de noms communs déterminés par l'article défini. Encore faut-il distinguer entre plusieurs cas. Et commencer par éliminer de cette classe les noms tels que La Flèche, La Grange, La Merluche, La Montagne, La Ramée, La Rapière, La Rivière, La T(b)orillière ${ }^{7}$, La Violette et

${ }^{3}$ Ces chiffres ne correspondent pas à ceux qui sont fournis par le site www.etudes françaises.net/nef base/moliere. Cette divergence s'explique essentiellement par le fait que l'inventaire du site est celui des personnages, et le mien celui des noms propres. Je signale en outre que la liste du site oublie l'Alcidor des Fascheux.

${ }^{4}$ Le moins insignifiant de ces personnages anonymes est celui qui, dans la liste des «Acteurs » de Tartuffe est désigné par l'expression «Un exempt», qui ne saurait d'aucune façon être tenue pour un nom propre. Il est vrai que dans la rubrique de la «Dernière scène », la seule où il apparait, il est désigné par l'expression définie "L'exempt», qui le met sur le même pied que les personnages énumérés en 2.1.2.

${ }^{5}$ Les trois pièces examinées ici s'éloignent peu de cette moyenne : George Dandin a 8 personnages, Le Misantrope en a 11, Le Bourgeois gentilhomme 13, à condition de ne tenir compte que de ceux qui ont un nom propre, c'est-à-dire d'exclure ceux qui sont désignés par une expression non déterminée du type de «Élève du Maistre de Musique » ou « Garçon Tailleur ».

${ }^{6}$ On verra cependant, plus bas que je pose la question pour les deux Dorimène : celle du Mariage forcé et celle du Bourgeois gentilhomme.

${ }^{7}$ Dans l'édition de 1682, première édition de L'impromptu de Versailles, la graphie de ce nom ne comporte pas $\mathrm{d}^{\prime}-b-$ dans la liste des personnages, mais en trouve une dans le texte. 
L'Espine. Pour ceux-là, l'article est intégré au nom propre, comme pour les noms de villes tels que Le Mans ou Le Havre (dont le comportement morpho-syntaxique n'est pourtant pas exactement identique). La Grange a une variante De la Grange, qui indique clairement l'intégration totale de l'article au nom propre. L'ensemble de ces noms doit donc être versé dans la classe 2.3.2, qui se caractérise par son apparence patronymique. On sait d'ailleurs que deux d'entre eux au moins ( $L a T(b)$ orillière et (de) La Grange) ont été dans l'univers mondain d'authentiques patronymes ${ }^{8}$ : ceux de comédiens de la troupe de Molière. - Un détail au passage, que je livre sans commentaire : dans cette sous-classe de noms propres, le genre féminin est le seul à être représenté, même si tous les personnages désignés sont des hommes...

Restent les cas où l'article défini fonctionne bien comme tel. On l'observe dans les conditions suivantes :

2.1.1. Accèdent au statut de personnage des entités dont l'humanisation est dûment signalée par un nom qui, introduit par l'article défini et muni d'une majuscule, prend le statut de nom propre: c'est le cas pour La Comédie, La Musique et Le Ballet dans L'Amour médecin, L'Amour dans Psiché et La Nuit dans Amphitryon. Le Zéphire et Le Dieu d'un flewve de Psiché ainsi que La Statue du Commandeur de Dom Juan représentent des cas voisins.

2.1.2. Plusieurs titres prennent, toujours sous l'effet de l'article défini, le statut de nom propre. Sauf dans les deux cas de La Princesse d'Élide et de La Comtesse d'Escarbagnas dans les pièces dont elles sont les éponymes, le titre déterminé par l'article constitue à lui seul le nom propre du personnage, dont le patronyme ou la désignation nobiliaire restent non dits. C'est le cas pour Le Commissaire dans L'Avare, Le Comte et Le Vicomte dans La Comtesse d'Escarbagnas, Le Marquis dans la Critique de l'École des femmes, L'Opérateur dans L'amour médecin, Le Roy dans Psiché, Le Sénateur dans Le Sicilien.

2.2. Prend également le statut de nom propre le nom Maistre, sans article, dans deux types de distributions et avec deux sens différents. Dans L'Avare il introduit un prénom: Maistre Jacques et Maistre Simon. Dame, dans l'unique Dame Claude, est son équivalent féminin. Dans Le Bourgeois gentilhomme, il désigne les praticiens de différents métiers, transformant en nom propre l'énonciation de leur profession : Maistre à dancer, Maistre d'armes, Maistre de musique, Maistre de philosophie, Maistre Tailleur.

2.3. Certains noms propres moliéresques présentent une ressemblance plus ou moins forte avec les noms propres de l'univers mondain de l'époque. On distingue les cas suivants :

${ }^{8}$ Je n'aborderai pas ici le problème du statut éventuellement pseudonymique de certains de ces noms : on apercevra plus bas que le nom de Moliere, «authentique » pseudonyme, est aussi le nom propre d'un personnage de L'Impromptu de Versailles. 
Michel Arrivé

2.3.1. Le nom propre se trouve réduit au seul prénom. Il est le plus souvent bien français, avec pas mal d'hypocoristiques morphologiquement divers. Mais il y a aussi plusieurs prénoms d'origine étrangère, espagnole ou italienne, voire arabe. Je les énumère, sans faire ces distinctions à peu près évidentes, par ordre alphabétique. On rencontre ainsi Agnès, Alain, Albert, André, Angélique, Anselme, Armande, Carle, Cathau et Cathos, Charlotte, Claudine, Colin, Corine, Dorine, Élise, Elvire, Enrique, Flipote, Francisque, Georgette, Géronimo, Gros-René, Gusman, Hali, Henriette, Hiacinte (qui, dans Les Fourberies de Scapin, est une femme: cas non unique de prénom épicène), Horace, Hypolite (personnage féminin de L'Étourdi), Ignès, Isabelle, Isidore (encore une femme, dans Le Sicilien), Jacqueline, Jeannot, Julie, Julien, Léonor, Lisette et Lysette, Lonison, Lucas, Lucette, Lucile, Magdelon, Mariane, Marinette, Marotte, Martine, Matburine, Nicole, Octave, Périn, Pierrot, Sabine, Silvestre, Thibaud, Toinette et Zerbinette. Pernelle est le seul prénom - s'il est authentiquement tel - à être présenté par le titre Madame. Les prénoms espagnols - francisés ou non - Alonse, Alphonse, Carlos, Juan, Louis et Pedre sont précédés par le titre Dom.

2.3.2. Le nom se réduit à un patronyme. J'entends par là une forme susceptible d'être interprétée comme un patronyme vraisemblable. On observe d'une part les cas de Béjart et Brécourt (noms de comédiens de la troupe de Molière), d'autre part les noms en La signalés au début de la rubrique 2.1, ainsi qu'un certain nombre d'autres noms : Du Bois, Du Croisy, Jodelet, Moliere (qui, on s'en souvient, est un personnage de L'Impromptu de Versailles). On peut ajouter à cette liste les noms de Mascarille, Tartuffe, Trissotin, Trufaldin et Vadius, que leur aspect formel et leur étymologie plus ou moins transparente peuvent faire tenir pour des sobriquets diversement désobligeants. Mais n'est-ce pas le cas pour un grand nombre de patronymes, eux aussi originellement sobriquets? On peut aussi songer à les renvoyer à la classe 2.4.

2.3.3. Toujours réduit au patronyme, le nom est précédé selon le sexe et, pour les femmes, selon l'âge ou le statut social par les titres Monsieur, Madame et Mademoiselle. Si on ne dénombre pas moins de vingt Monsieur + patronyme ${ }^{9}$, il n'y a que deux Madame + patronyme (le patronyme est dans les deux cas - Madame de Sotenville et Madame Jourdain - celui de l'époux). Les Mademoiselle + patronyme sont au nombre de six. Ces six noms sont d'authentiques noms propres mondains. On peut ajouter à cette liste les trois Dom introduisant un nom propre qui semble être plutôt un patronyme qu'un prénom : Dom Alvar, Dom Garcie et Dom Lopes.

2.3.4. Restent deux cas: ceux de George Dandin et de Thomas Diafoirus. Ils constituent une exception remarquable dans l'onomastique moliéresque: l'énonciation cumulée du prénom et du patronyme. C’est dire l'intérêt que

\footnotetext{
${ }^{9}$ Le Monsieur Guillaume de L'amour médecin est le seul à pouvoir être interprété comme un prénom ou un patronyme. Les noms des médecins de L'amour médecin relèvent de la classe 2.4.1.
} 
présentent ces deux noms, et surtout le premier, qui donne son titre à la pièce où il apparait ${ }^{10}$.

2.4. Un grand nombre de noms appartiennent à un inventaire spécifique, réservé aux personnages de théâtre. Ces noms ont la particularité d'échapper aux règles de l'onomastique mondaine: ils ne sont ni prénoms, ni patronymes, mais désignations uniques du personnage. Celui-ci peut d'ailleurs, dans son éventuelle existence mondaine en dehors de la pièce, porter un autre nom, conforme aux règles habituelles. Le cas d'une telle évocation, à vrai dire très paradoxale, est à ma connaissance unique dans l'œuvre de Molière. On la trouve dans la bouche de Lubin à propos du Clitandre de George Dandin:

C'est le Seigneur de nostre pays, Monsieur le Vicomte de chose... Foin je ne me souviens jamais comment diantre ils baragouynent ce nom là, Monsieur Cli... Clitande (sic) (I, 2, p. 26).

Ainsi Clitandre n'est que le nom du personnage sur la scène. Il a dans la vie « réelle » qui lui est ici fugitivement prêtée un autre nom : «le Vicomte de chose », où chose n'est pas le substitut de Clitandre - *le Vicomte de Clitandre est une expression totalement impossible dans le discours théâtral de l'époque - mais du nom nobiliaire du personnage, qui ne sera jamais énoncé. On remarquera qu'on ne sait pas trop quel est, de ces deux noms, celui dont le pauvre Lubin dit ne pas se souvenir: car il a oublié les deux, le premier totalement, le second partiellement.

Appartiennent à cette classe un grand nombre de noms moliéresques. Pour quelques-uns on peut hésiter entre les classes 2.3 .1 ou 2.3.2 et la classe 2.4. Plusieurs critères peuvent intervenir. La pièce dans laquelle ils apparaissent lève parfois l'ambiguité : l'Arsinoé et l'Éliante du Misantrope ne peuvent guère être - compte tenu du statut des autres noms propres de la pièce - que des noms spécifiquement théâtraux, même s'il n'est pas impossible que ces noms aient pu être à l'époque utilisés comme prénoms. On se souvient d'ailleurs qu'Arsinoé, nom de plusieurs princesses macédoniennes et égyptiennes, est aussi celui de la « seconde femme de Prusias » dans le Nicomède de Corneille, qui date de 1651 et a été joué par la troupe de Molière en 1658. Béline ni Bélise pas plus que Nérine ne sont des prénoms très vraisemblables. Criquet, Galopin et Basque (selon le nom de sa province d'origine) sont des sobriquets traditionnellement donnés à des laquais. Covielle a évidemment perdu le statut de diminutif qu'avait en italien son étymon Jacoviello. Mais Lubin est, dans le Francion de Sorel (p. 167), le nom (sans doute le prénom, car il y a un Saint Lubin...) d'un paysan chapardeur et naïf qui ressemble fort à son homonyme de George Dandin. Dans des registres différents, Gorgibus - dont le sort, dans La Jalousie du Barbouillé, évoque celui de George Dandin - et Marphurius tout comme les trois formations italianoïdes en $S+$ consonne : Sbrigani, Scapin, Sganarelle semblent échapper à la fois au statut du prénom et à celui du patronyme.

${ }^{10}$ Autre témoignage du caractère exceptionnel de ce type de nom propre : le cas de Lucile dans Le bourgeois gentilhomme: Lucile, fille de Monsieur Jourdain et de Madame Jourdain est bien Lucile Jourdain, mais elle n'est jamais dénommée ainsi dans la pièce. 
Michel Arrivé

Il est hors de question d'étudier ici en détail tous ces noms. Je me contente de formuler deux remarques :

2.4.1. Un grand nombre d'entre eux affichent plus ou moins clairement une étymologie latine (Célie, Lélie - le premier féminin, le second masculin dans L'Étourdi, décidément riche en cas d'ambiguité sexuelle ${ }^{11}$-, Lucinde, Valère) et, surtout, grecque. Beaucoup de ces derniers sont motivés de façon transparente - pour qui sait le grec - par rapport au personnage qu'ils désignent : dans des directions différentes, on peut citer du côté positif Ariste ( l'excellent »), Philinte ("l'amical») et Clitandre ("l'homme illustre»), du côté négatif Harpagon («le pillard») ou la série très désobligeante des noms des médecins de L'Amour médecin. Certains sont rétifs à toute étymologie vraisemblable : en quoi Orontequi est le nom d'un personnage de Xénophon - est-il spécialement « voyeur »? D'autres noms ont pour étymons des personnages plus ou moins illustres de la mythologie, de l'histoire ancienne ou, plus rarement, médiévale : ainsi Pandolfe a été le nom, aux alentours de l'an mil, de plusieurs princes successifs de Bénévent et de Capoue.

2.4.2. De façon peut-être un peu moins patente, quelques noms de cette classe sont d'origine ou d'apparence arabe. Le plus évident est l'Almanzor des Précieuses ridicules. Passé par un roman de Gomberville, Polexandre, ce nom - qui signifie en arabe «le victorieux » - a été porté, lui aussi aux alentours de l'an mil, par un prince musulman d'Andalousie. Il est donné, par antiphrase, à un petit laquais qui se contente, dans la pièce, d'énoncer un obéissant "Madame » adressé à Magdelon. Alcantor dans Le Mariage forcé et Alcidor dans Les Fascheux peuvent au moins être pris pour araboïdes, en raison de leur $A l$ - initial, de leur finale en or et, pour le second, de l'élément -cid-, qui ne peut manquer de faire penser au héros cornélien, dont on connait l'étymon. Quant à Hali, il a déjà été recensé en tant que prénom.

Me laisserai-je aller, après ce bref effort de classification, à quelques interrogations diversement aventureuses ? En voici quelques-unes.

1. Se pourrait-il que s'observent dans cet inventaire de noms propres certaines régularités morphologiques ? Je ne fais que poser une question, qui porte de la même façon sur quatre séries de faits : l'élément $S$-devant consonne, commun, on l'a vu plus haut, aux trois noms Sbrigani, Scapin et Sganarelle fonctionne-t-il dans le lexique étudié comme son homophone le préfixe $s$ - de l'italien? Autrement dit, cet élément commun aux trois noms marque-t-il un trait commun aux trois personnages ${ }^{12}$ ? Et lequel ? La même question se pose pour l'élément -olphe/olfe, commun à Arnolphe et Pandolfe $e^{13}$,

11 Dans la même pièce Hypolite est une femme.

12 À supposer qu'ils ne soient que trois : on sait que le problème se pose pour Sganarelle.

${ }^{13}$ Est-il possible d'ajouter à ces deux noms celui, très proche, de Panulphe ? On sait que c'était le nom, lors de la première représentation, en 1667 , du personnage qui devait ensuite prendre le nom de Tartuffe. 
pour l'élément -ine, qui apparait dans dix noms féminins (Béline, Claudine, Corine, Dorine, Frosine, Jacqueline, Martine, Mathurine, Nérine, Sabine) et son pendant masculin -in, qu'on trouve dans treize noms masculins (Colin, Galopin, George Dandin, Lubin, Monsieur Filerin, Monsieur Harpin, Ormin, Périn, Ragotin, Scapin, Trissotin, Trufaldin, Villebrequin, sans parler d'Alain ni de Monsieur Jourdain). Autrement dit, ces éléments fonctionnent-ils dans les noms qu'ils affectent comme des suffixes, marques de traits communs aux personnages désignés ?

2. Se pourrait-il qu'aient un sens - et lequel ? - les diverses bizarreries qu'on a notées quant aux relations entre genre des noms et sexe des personnages? Ainsi on a vu que tous les personnages désignés par un patronyme en La sont des hommes. On observe d'autre part de nombreux cas de prénoms généralement masculins appliqués à des femmes : ainsi Hiacinte, Hypolite et Isidore sont des femmes. De très nombreux noms ne laissent rien prévoir du sexe de la personne désignée : Aglante, Aglaure, Egiale, Cidippe et Phaìne sont des femmes. Inversement Lélie et Cléomène sont des hommes. On a compris que je réserve un examen spécifique au plus spectaculaire de ces cas : le nom d'Alceste, attribué à un homme en dépit d'une illustre et très ancienne tradition.

3. N'est-il pas possible, devant certains noms en $A l$ - d'hésiter entre une origine grecque et un étymon arabe ? Dans certains cas, la décision est possible. Ainsi Alcidas est grec : c'est la forme dorienne d'Alcidès, c'est-à-dire Héraclès. Mais on a vu qu'Alcidor ${ }^{14}$ présente les traits - au moins moliéresques - de l'arabité. En tout cas on ne connaît dans la Grèce antique aucun Alcidor. Alcandre ${ }^{15}$ est, entre autres, un personnage de Plutarque. Mais aucun Alcantor n'est connu en Grèce. La forme du mot évoque de façon précise certains noms propres arabes hispanisés (par exemple la ville d'Alcantara). - On a sans doute compris que j'en viens à me poser la question pour Alceste : son nom, décidément bien problématique, est-il aussi assurément grec que le répètent les notes de toutes les éditions ?

Après ces remarques générales, j’aborde maintenant les questions spécifiques qui m'ont incité à entreprendre cette recherche.

\section{George Dandin}

Ce n'est pas le patronyme qui m'intéresse le plus. Il a pour étymon peu discutable l'adjectif dandin, qui recevra dans le Dictionnaire de Richelet - en 1680 - les définitions suivantes: «Sorte de sot et de niais qui va regardant çà et là », puis «manière de benet et de lourdaud qui a un air languissant et innocent ». Mais dès 1606 Nicot donnait, si j’ose dire, une définition de même tabac : «Celui qui baye çà et là par sottise et badaudise sans avoir contenance arrestée ». On sait que ce patronyme avait

\footnotetext{
${ }^{14}$ Un Alcidor apparaît dans Les Bergeries de Racan.

15 On se souvient aussi qu'Alcandre est dans L'illusion comique (1639) le nom du Magicien. Rotrou avait lui aussi utilisé ce nom pour un Magicien dans La Bague de l'Oubli et L'Innocente Infidélité.
} 
déjà connu, avant Molière, la fortune littéraire: dans le Tiers Livre de Rabelais, le chapitre XLI met en scène «l'appointeur de procès » Perrin Dendin (sic). Quelques mois après George, Perrin resurgira dans Les Plaideurs de Racine. Et La Fontaine le fera réapparaître, en 1671, dans la fable de «L'huître et les plaideurs ». Dans la pièce de Molière, le nom est pompeusement anobli sous la forme de la Dandinière. Type de manipulation du patronyme qui n'est pas sans exemple dans l'onomastique mondaine de l'époque ${ }^{16}$.

En dépit des apparences, c'est sans doute le prénom qui est le plus intéressant. On peut, certes, en rappeler, avec Bernadette Rey-Flaud ${ }^{17}$ l'étymon rural, qui s'accorde pleinement avec l'origine paysanne de George: il la rappelle à diverses reprises, notamment dès le monologue inaugural de la première scène. Mais on n'a - en tout cas dans ce que j'ai lu - point aperçu la structure à la fois phonique et graphique de ce prénom : l'élément -OR- sépare deux occurrences du phonème / $/ \mathrm{m}$ marqué par le digramme GE. L'absence de l'-s finale - conforme, j'en conviens aux mœurs de l'époque ${ }^{18}$ - rend cette structure parfaitement visible, c'est-à-dire lisible :

$$
\text { GE - OR - GE }
$$

En somme, George, c'est le sujet - le je qui parle, enfin, qui essaie de parler divisé, au sens le plus matériellement littéral du mot, par l'or. Car c'est la possession de l'or qui a entraîné son mariage avec la très antiphrastique Angélique et, par là, les horribles problèmes que rencontre le triste George. Il en vient à douter de sa propre identité : est-il devenu noble, comme semble l'indiquer la ridicule expansion de Dandin en de la Dandinière? Ou est-il resté paysan ? L'interrogation le perturbe de façon constante. Le je qui parle, ai-je dit? Mais précisément George a des difficultés avec le je. Difficultés spécifiques: c'est un fait que les problèmes de désignation liés à sa situation sociale et familiale sont pour lui toujours ardus. Madame de Sotenville estelle « sa belle-mère » ? Que non pas ! Elle le lui fait remarquer avec sévérité :

Ne vous déferez-vous jamais avec moy de la familiarité de ce mot de ma belle-mère, \& ne sauriez-vous vous accoustumer à me dire Madame [?] (I,4, p. 30).

Question plus grave encore : Angélique est-elle «sa femme » ? Cette fois, c'est M. de Sotenville - le beau-père - qui lui interdit de désigner Angélique par l'expression ma femme:

Tout beau! Aprenez aussi que vous ne devez pas dire ma femme quand vous parlez de nostre fille.

D'où la colère stupéfaite de George :

\footnotetext{
${ }^{16} \mathrm{Il}$ en subsiste nécessairement quelques traces dans l'onomastique contemporaine. Ainsi Le Monde du 18 juin 2002 nous apprend, dans la nécrologie du Comte Jean de Beaumont, qu'il se nommait Bonnin de la Bonninière.

${ }^{17}$ L'auteur (1991) se livre à quelques réflexions sur l'étymologie - $\gamma \varepsilon o \rho \gamma 10 \varsigma-$ de George, qui affiche le côté paysan du personnage et sa relation avec le farcesque George Le Veau.

${ }^{18}$ En somme, Molière n'a pas eu à supprimer une $-s$ déjà absente : l'orthographe de l'époque lui offrait, tout prêt, le seul nom que son personnage pût porter.
} 
J'enrage. Comment, ma femme n'est pas ma femme ? (I, 4, p. 31-32).

Conformément aux habitudes typographiques de l'époque, la différence n'est pas marquée entre l'usage mondain de l'expression et sa mention autonymique. C'est bien pourtant, de toute évidence, ce problème qui est posé à George : comment doit-il désigner la personne qu'il a épousée? Et quel est au fait, dans l'expression proscrite, l'élément qui détermine l'interdiction? La désignation à laquelle recourt le beau-père nostre fille - montre que, plutôt que l'innocent substantif femme, c'est le possessif de première personne $\boldsymbol{m a}$ qui est interdit.

C'est en effet avec la première personne, c'est-à-dire sa propre désignation par je que $G e-O r-G e$ a les plus graves problèmes. Aussi en vient-il très souvent à renoncer à l'effort de parvenir jusqu'à ce je difficile d'accès. Il se contente d'un vous cérémonieux, qui apparât immédiatement après l'occurrence redoublée de son nom, comme si le je interdit - au même titre que le ma de ma femme - était à la fois compensé et rappelé par la répétition vide de sens de son propre signifiant, écartelé par la mention de l'or:

George Dandin, George Dandin, vous avez fait une sotise (sic) la plus grande du monde (I, 1, p. 22 ; voir aussi les monologues qui constituent, pp. 28-29, puis 49, les scènes 3 et 7 de l'Acte $\left.\mathrm{I}^{19}\right)$.

En un point du texte le vous fait place à un tu plus familier. Dans des conditions spectaculaires: Dandin passe du $t u$ au vous dans la même réplique, avant de parvenir enfin à un laborieux $j e$ :

Ah, George Dandin, où t'es-tu fourré ? Et de grâce, mettez pour un moment vostre gentilhommerie à costé, \& souffrez que je vous parle maintenant comme je pourray ${ }^{20}$. Au diantre soit la tyrannie de toutes ces histoires-là ! Je vous dy donc que je suis mal satisfait de mon mariage (I, 4, p. 32).

On aura remarqué que cette difficile progression vers le je s'observe précisément après l'interdiction qui lui est faite de désigner Angélique comme « ma femme ».

\section{Les personnages du Bourgeois gentilbomme}

On vient d'apercevoir que George Dandin est le sujet divisé par l'or. Qu'en est-il donc des personnages qui, dans Le Bourgeois gentilhomme, portent aussi un nom où s'affiche l'or?

2.1. Pour D-or-ante et D-or-imène, peu de difficultés : les deux personnages ne pensent qu'à l'or, n'agissent que pour le conquérir. C'est ce qui est signifié de la façon

\footnotetext{
${ }^{19}$ Dans cette dernière, un je timidement tenté par Dandin avorte prématurément et fait place immédiatement au vous.

20 On remarquera l'ambiguiité du vous de cette réplique : s'adresse-t-il à Dandin lui-même ? Ou à l'horrible couple de ses beaux-parents? On remarquera que le et de l'édition originale appuie la première lecture. Il est corrigé en $e h$ ! par plusieurs éditions modernes qui lisent sans doute de la seconde façon.
} 
la plus explicite par la scène II de l'Acte V. Leur nom est donc constitué par lui. Dorante lui confère la forme d'un participe actif, Dorimène celle d'un participe passif: c'est du moins ce que livre la plus élémentaire des grammaires grecques. Et c'est ce qui correspond au rôle des deux personnages: Dorante essaie - activement, oh! combien! - d'acquérir l'or, Dorimène en est, passivement, la destinataire. À moins, naturellement, qu'on ne songe qu'elle paie par son mariage avec Dorante l'or qu'il a enfin, qu'elle croit ou feint de croire qu'il a :

J'ay veu là des aprests magnifiques, \& ce sont des choses, Dorante, que je ne puis plus souffrir. Oüy, je veux enfin vous empécher vos profusions ; \& pour rompre le cours à toutes les dépenses que je vous voy faire pour moy, j’ai résolu de me marier promptement avec vous. C'en est le vrai secret, $\&$ toutes ces choses finissent avec le Mariage (V, 2, p. 258).

On le voit, au sens le plus littéral du terme : le nom de Dorimène peut aussi se lire D - OR - HYMEN.

Une confirmation de cette analyse pourrait sans doute être fournie par l'autre Dorimène, celle - précisément - du Mariage forcé : pour rassurer son amant Lycaste, qui s'inquiète de la voir épouser Sganarelle, la jeune personne ne s'embarrasse pas de précautions inutiles :

$[\ldots]$ ce Mariage ne doit point vous inquiéter. C'est un Homme que je n'épouse point par amour ; \& sa seule richesse me fait résoudre à l'accepter. Je n'ay point de bien. Vous n'en avez point aussi ; \& vous sçavez que sans cela on passe mal le temps au Monde, \& qu'à quelque prix que ce soit, il faut tâcher d'en avoir (sc. 7, p. 108).

Un peu plus brutale encore, un peu plus cynique s'il se peut, cette première Dorimène - car Le Mariage forcé est antérieur de six ans au Bourgeois gentilhomme - présente tous les traits de la seconde, et mérite autant qu'elle son nom.

2.2. Pour Monsieur Jourdain, les données littérales sont un peu moins transparentes. Il convient d'abord, après Serroy, 2000, p. 14, de noter que son patronyme rime avec celui de George Dandin. Et on peut tomber d'accord avec Serroy pour noter qu' » en matière de patronyme et de prétention sociale il est exactement à [son] opposé ». C'est vrai : Dandin regrette son accès - à vrai dire imparfait - à la "gentilhommerie », Jourdain fait porter tout son désir vers cet accès. Mais il est un point commun entre les deux personnages : la possession de l'or, seul moyen de parvenir au statut convoité ou redouté sitôt acquis. Se pourrait-il qu'il fût absent du nom de Monsieur Jourdain ? Il faut ici prendre garde au fait que Jourdain - s'il n'a pas de prénom - a un autre nom : Jordina. Il le dit lui-même, quand il fait à son épouse le récit de la Cérémonie Turque qui l'a élevé au rang de Mamamouchi:

$$
\begin{aligned}
& \text { Monsieur Jourdain. - Mahameta per Jordina. } \\
& \text { Madame Jourdain. - Qu'est-ce que cela veut dire? } \\
& \text { Monsieur Jourdain. - Jordina, c'est-à-dire Jourdain (V, 1, p. 256) }
\end{aligned}
$$

On le remarque: Jourdain adopte comme sien le nom Jordina et corrige la forme inexacte que le Mufti a donnée à son nom, dans la Cérémonie Turque qui vient de s'achever: «Mahameta per Giourdina ». Son vrai nom, ce n'est pas Giourdina, c'est 
bien Jordina. Et l'on constate alors que son nom se lit J - OR - DINA, avec la même infixation du segment - or-que dans le prénom de GE - OR - GE. La différence entre les deux personnages tient dans le fait que le pronom je reste, pour le «PalaDIN" qu'est devenu J- OR - DINA, intact. Il est même majestueusement étoffé par l'expansion doublement prestigieuse - l'OR et la dignité de PalaDIN - qui lui est conférée. Aussi voit-on que Jourdain n'éprouve nullement les difficultés de Dandin pour accéder au statut de sujet : il dit je, constamment.

\section{Alceste et Célimène}

Ici les problèmes se compliquent. Et les solutions que j'envisage risquent, j'en conviens, de paraitre quelque peu alambiquées. Elles reposent, de la même façon que celles qui viennent d'être envisagées, sur l'hypothèse que le nom propre moliéresque est signifiant non seulement dans son ensemble, mais aussi par ses segments. Pas nécessairement tous ses segments : sa grammaire interne n'est pas exactement celle de la langue. On a vu par exemple que dans Dorimène, le $D$ - initial reste, sans doute, non signifiant, à la différence des deux autres éléments. Quoi qu'il en soit, cette signifiance est souvent occultée par le fait que le nom propre conserve sa fonction habituelle de désignateur apparemment arbitraire. De ce fait on oublie souvent de le lire comme le texte - le texte en miniature - qu'il est aussi.

Je commence par le moins opaque de ces microtextes: autant que dans Dorimène, il y a de l'bymen dans Célimène. Comme plusieurs critiques l'ont aperçu, son nom condense ceux de la Célie de L'Étourdi et de la Climène du Sicilienn ${ }^{21}$. C'est cette condensation qui fait apparaitre l'bymen dans son nom. Hasard de la répartition des finales entre les personnages féminins? Célimène elle-même nous détrompe, dans un passage décisif de la pièce. Il faut le lire à la lettre, au sens le plus littéral du mot lettre. Au moment où, dans la dernière scène, se précise le projet d'Alceste de se retirer dans son «Désert », elle consent, pour l'en détourner, à lui proposer le mariage, mais un mariage tel qu'il n'entraîne point pour elle la «Solitude » absolue qu'envisage son Amant :

Si le Don de ma main peut contenter vos vœux, Je pourrai me résoudre à serrer de tels Nœuds ; Et l'Hymen...

Alceste ne la laisse même pas terminer sa phrase :

Non, mon Cœur, à présent vous déteste,

Et ce refus, luy seul, fait plus que tout le reste. (V, 4, vers 1178-1181, p. 26).

Qu'en est-il exactement de cette brutale rebuffade, qui entraine immédiatement le départ de Célimène? Elle est d'une rigueur extrême: car elle n'entend pas, littéralement, ce qu'il y a de positif dans la réponse de Célimène : l'acceptation, mieux,

${ }^{21}$ Une autre Climène apparait dans La Crique de l'École des femmes et une Clymène dans Les Fâcheux. On sait aussi que Célimène a été préalablement l'héroïne, en 1633, d'une comédie de Rotrou à laquelle elle donne son titre : La Célimène. 
la proposition du mariage. Non, Alceste n'entend qu'une chose : Célimène vient d'énoncer son propre nom, amputé, il est vrai, de son premier phonème : son Et l'hymen, c'est Célimène le $C$ - en moins, ou le /s/ si l'on tient à l'aspect phonique. Il y a donc un manque dans sa proposition, manque littéral qui, sitôt reconnu, entraine le refus absolu d'Alceste. Célimène, Alceste la veut toute, comme femme et comme nom. Et la perte que subit son nom répète, de façon insupportable, ce qu'a d'incomplet sa proposition désormais détestable.

D'autant que la lettre perdue apparait aussi dans son propre nom : le -c- , alias /s/, est également présent dans AL - C - ESTE. J'entends l'objection : il n'y est point dans la même position. Voire! C'est ici qu'il faut reprendre le problème de l'étymon du nom d'Alceste.

Comme on sait, ce nom est originellement féminin dans la tradition dramatique : Alceste est dans le mythe antique et dans la tragédie d'Euripide le nom de la femme d'Admète. Elle accepte de mourir à sa place, avant d'être opportunément ramenée des Enfers par Héraclès. Quel rapport entre le sort de cette femme et celui du Misantrope ? Faut-il essayer de trouver à l'Alceste de Molière des traits féminins ? Et spécifiquement des traits alcestiens, au sens tragique du terme ? J'avoue que j'ai été tenté d'y songer. Mais j’ai renoncé : il faudrait des trésors d'ingéniosité pour repérer quoi que ce soit de féminin et plus encore d'euripidien dans le personnage. À ma connaissance, personne n'a entrepris cet effort inutile. Serait-ce alors que tout souvenir de l'Alceste d'Euripide est effacé du Misantrope? Que non pas. Pour une raison textuellement irréfutable. Un Acaste, «marquis » de son état, apparait dans la pièce: jusqu'au dénouement, il n'est guère qu'une pâle réplique du prestigieux Clitandre. Mais il prend une place déterminante dans la dernière scène : c'est lui qui donne lecture du très désobligeant billet de Célimène, dont la révélation va précipiter le dénouement. Or il se trouve qu'Acaste est, dans le mythe antique le frère d'Alceste. Dans la tragédie, il n’apparaît pas comme personnage, mais est cité par Phérès, père d'Admète, qui regrette qu'il ne semble point songer à venger la mort de sa sœur. Quelle peut être la fonction dans le Misantrope de cette rencontre onomastique du frère et de la sœur? On ne peut guère l'envisager que de façon négative: la présence d'Acaste - et son intervention déterminante lors du dénouement de la pièce rappellent à la fois l'existence du mythe d'Alceste et sa non-pertinence à l'égard du personnage qui porte son nom.

Faut-il donc renoncer à l'étymologie grecque d'Alceste? Les annotateurs ne s'y résolvent généralement pas, et vont exhumer l'adjectif alkestès, hapax signifiant «fort», chez l'obscur et très tardif Oppien ${ }^{22}$. Pour ma part, je me résoudrais volontiers à cet abandon. Mais qu'on se rassure: je n'ai pas dans ma manche un étymon substitutif arabe. Je remarque simplement que l'élément $A l$-, du seul fait de sa fréquence à l'initiale dans les noms moliéresques, peut sans difficulté s'isoler de la suite du nom qu'il préfixe, comme l'article qu'il est étymologiquement peut s'effacer du nom de l'Alcoran, qui est explicitement cité, sous cette forme, dans la «Cérémonie turque » du Bourgeois gentilhomme (IV, 5, p. 252). Reste donc, pour notre personnage dépouillé de son $A l$ - initial, l'élément -ceste.

${ }^{22}$ La plupart d'entre eux rappellent aussi qu'un Alceste apparaît dans un roman - Polyxène - d'un autre Molière, Molière d'Essertines. 
Rien ne permet d'affecter raisonnablement un sens à ce segment -ceste. Le mot existe, certes : c'est le gantelet de cuir utilisé par les athlètes antiques dans le pugilat. Trop brutal, tout de même, pour Alceste, dont les diverses foucades restent - non sans effort de sa part: qu'on songe à la fin de la scène du sonnet, où il s'en faut de peu qu'il n'en vienne aux mains avec Oronte - verbales. -ceste fait donc sans doute partie de ces éléments non signifiants qui, on l'a aperçu, se rencontrent dans plusieurs noms propres moliéresques. Non signifiant ? Peut-être. Mais non dépourvu de fonction. Il exhibe à son initiale la lettre $c$-, celle-là même qu'il a en commun avec C-élimène. Et c'est, on l'a vu, l'effacement de cette lettre - le seul trait qu'il ait en commun avec elle - par Célimène elle-même qui détermine son retrait définitif, tout juste précédé par le départ de la femme aimée.

Les noms propres moliéresques ont une spécificité - qu'ils partagent, sans doute, avec de nombreux noms propres littéraires: ils ne se contentent pas de désigner, ils signifient. Mais selon des modes spécifiques : un peu à la façon dont les mots signifient dans le rêve. C'est dire la difficulté de leur analyse. Et les doutes dont s'accompagnent les spéculations littérales auxquelles on vient de se livrer.

Michel ARRIVÉ

Département des sciences du langage

Université Paris X

200, avenue de la République

92001 Nanterre cedex

michel.arrive@wanadoo.fr

\section{RÉFÉRENCES BIBLIOGRAPHIQUES}

1. L'édition de Molière qui a été utilisée - en raison de sa fidélité à l'orthographe des éditions originales - est celle de Gustave Michaut, Paris, Imprimerie Nationale, Collection Nationale des Classiques français, 1947, onze volumes. Le Mariage forcé se trouve au tome IV, Le Misantrope au tome V, George Dandin au tome VII et Le Bourgeois gentilhomme au tome VIII.

2. Les deux dictionnaires cités sont le Thrésor de la langue françoyse [...] de Jean Nicot, Paris, David Douceur, 1606 et le Dictionnaire françoys contenant les mots et les matières [...] de Pierre Richelet, Genève, Widerhold, 1680.

3. Les quelques œuvres littéraires citées ont été suffisamment identifiées dans le texte.

4. On n'a cité que deux articles: Rey-Flaud, Bernadette, 1991, «L'héritage de la farce médiévale dans le théâtre de Molière ", in Théatre et spectacle hier et aujourd'hui et Serroy, Jean, 2000, "Vous a-t-on point dit comme on le nomme?" Alceste, Dandin, Jourdain », Littératures classiques, 38, p. 9-17. 